\title{
Ibrutinib discontinuation in patients with relapsed or refractory chronic lymphocytic leukemia treated in a compassionate use program: A report from the Polish Adult Leukemia Study Group (PALG)
}

\author{
Elżbieta Iskierka-Jażdżewskaa ${ }^{1, C, D, F}$, Bartosz Puła 2,C,F , Agnieszka Szeremet ${ }^{3, B, F}$, Marek Hus ${ }^{4, B, F}$, Aleksandra Gołos ${ }^{2, B, F}$, \\ Jadwiga Hołojda ${ }^{5, B, F}$, Weronika Piszczek ${ }^{6, B, F}$, Paweł Steckiewicz ${ }^{7, B, F}$, Małgorzata Wojciechowska ${ }^{8, B, F}$, Jan Maciej Zaucha ${ }^{9,10, B, F}$, \\ Krzysztof Warzocha ${ }^{2, E, F}$, Krzysztof Jamroziak ${ }^{2, A, C, D, F}$ \\ ${ }^{1}$ Department of Hematology, Copernicus Memorial Hospital, Łódź, Poland \\ 2 Department of Hematology, Institute of Hematology and Transfusion Medicine, Warszawa, Poland \\ ${ }^{3}$ Department of Hematology, Blood Neoplasms and Bone Marrow Transplantation, Wroclaw Medical \\ University, Poland \\ ${ }^{4}$ Department of Haematooncology and Bone Marrow Transplantation, Medical University of Lublin, \\ Poland \\ ${ }^{5}$ Department of Hematology, Specialist District Hospital, Legnica, Poland \\ ${ }^{6}$ Department of Hematology, Copernicus Hospital, Toruń, Poland \\ ${ }^{7}$ Department of Hematology, Holycross Cancer Center, Kielce, Poland \\ ${ }^{8}$ Department of Hematology, Specialist District Hospital, Olsztyn, \\ Poland \\ ${ }^{9}$ Department of Oncological Propaedeutics, Medical University \\ of Gdańsk, Poland \\ ${ }^{10}$ Gdynia Oncology Center, Poland
}

A - research concept and design; B - collection and/or assembly of data; $\mathrm{C}$ - data analysis and interpretation;

$D$ - writing the article; $E$ - critical revision of the article; $F$ - final approval of the article

Address for correspondence

Elżbieta Iskierka-Jażdżewska

E-mail: elaiskierka@gmail.com

Funding sources

None declared

Conflict of interest

None declared

Received on December 19, 2017

Reviewed on May 24, 2018

Accepted on November 21, 2018

Published online on June 14, 2019

Cite as

Iskierka-Jażdżewska E, Puła B, Szeremet A, et al. Ibrutinib discontinuation in patients with relapsed or refractory chronic lymphocytic leukemia treated in a compassionate use program: A report from the Polish Adult Leukemia Study Group (PALG). Adv Clin Exp Med. 2019;28(8):1051-1057. doi:10.17219/acem/99911

DOI

10.17219/acem/99911

\section{Copyright}

Copyright by Author(s)

This is an article distributed under the terms of the Creative Commons Attribution Non-Commercial License (http://creativecommons.org/licenses/by-nc-nd/4.0/)

\begin{abstract}
Background. Development of a novel class of drugs, the B-cell receptor-signaling inhibitors, including ibrutinib, has been a major achievement in the therapy of refractory or relapsed chronic lymphocytic leukemia (CLL). However, the CLL patients who have discontinued the ibrutinib treatment in clinical trials have been reported to have poor prognosis.
\end{abstract}

Objectives. In this retrospective study by the Polish Adult Leukemia Group (PALG), we analyzed the reasons for ibrutinib cessation and outcomes after discontinuing ibrutinib in refractory or relapsed CLL patients treated in a compassionate use program in Poland.

Material and methods. Polish CLL patients were included if they discontinued ibrutinib for any reason. The clinical data on the course of ibrutinib treatment was collected anonymously using electronic Case Report Forms (CRFs). The causes of discontinuation of ibrutinib as reported by the treating physicians were analyzed.

Results. Thirty-seven patients who discontinued ibrutinib were identified. The median duration of ibrutinib treatment in this group was 4.4 months (range: 0.2-25.2). The main reason for discontinuing ibrutinib was adverse events $(n=20,54 \%)$, while 14 (38\%) patients discontinued therapy due to disease progression and $3(8 \%)$ due to other causes. The most common treatment complications that led to ibrutinib cessation were severe respiratory tract infections ( 9 patients, 24\%). In the group discontinuing ibrutinib for progressive disease, 11 patients progressed with untransformed CLL, while in 3 patients, a rare type of Richter transformation to Hodgkin's lymphoma was diagnosed. Twenty-nine patients (78\%) died during the follow-up period, and median overall survival (OS) reached 2.0 months $(95 \% \mathrm{Cl}=0.8-5.5$ months). Importantly, no significant survival difference was detected between patients who discontinued ibrutinib due to disease progression and due to adverse events.

Conclusions. The results of this analysis indicate that ibrutinib discontinuation in relapsed or refractory CLL is associated with poor prognosis regardless of the reason for ibrutinib cessation.

Key words: tyrosine kinase inhibitor, chronic lymphocytic leukemia, ibrutinib

Acknowledgements

This study was supported by the Polish Adult Leukemia Study Group (PALG). We would like to acknowledge the following clinicians for providing us with information about patients used in our study: Michał Osowiecki MD, PhD; Wanda Knopińska-Posłuszny MD, PhD; Daria Zawirska MD, PhD; Beata Kumiega MD, PhD; Janusz Hałka MD, PhD, Anna Waszczuk-Gajda MD; PhD. We also acknowledge Paulina Wieszczy for her help with statistical analysis. 


\section{Introduction}

Chronic lymphocytic leukemia (CLL) is characterized by clonal proliferation and accumulation of mature B-cells, typically co-expressing CD5 and CD23, within the blood, bone marrow, lymph nodes, and spleen. ${ }^{1}$ The disease primarily occurs in elderly patients and has a very heterogeneous course. ${ }^{2}$ According to current guidelines, patients with an inactive, early stage CLL (Binet A/B or Rai 0-II) should undergo active surveillance, while therapy needs to be started in patients with advanced or active disease. ${ }^{3}$

Until recently, the standard treatment approach to CLL was based on anti-CD20 antibody-containing immunochemotherapy. However, even if prolonged remissions could be initially obtained in a proportion of patients, eventually the disease develops refractoriness to such therapy. Major progress in treating relapsed or refractory CLL has recently been achieved by the development of a novel class of drugs: B-cell receptor signaling inhibitors, including ibrutinib (Imbruvica; Janssen Cilag, Beerse, Belgium). Ibrutinib is a first-in-class, oral, once-daily therapy that inhibits Bruton's tyrosine kinase (BTK). ${ }^{4}$ This kinase is a member of the Tec kinase family and a key molecule in the B-cell receptor signaling complex, playing an important role in the survival and spread of normal B cells. ${ }^{5}$ It was found that this kinase also affects the maintenance and expansion of various B-cell malignancies, including CLL. ${ }^{5,6}$

Clinical trials on ibrutinib in relapsed or refractory and treatment-naïve CLL patients have been very promising and led to the drug being approved in the USA and EU. ${ }^{4,7-10}$ Furthermore, data from several clinical trials has shown that ibrutinib is generally well-tolerated. ${ }^{4,8-10}$ The most common adverse events were mostly mild (grade 1-2). . $^{4-10}$ The prevailing ibrutinib-related adverse events included diarrhea, fatigue, cough, edema, upper respiratory tract infections, arthralgia, rash, fever, and mild hematological toxicity. Nonetheless, some unexpected adverse events have also been reported which included mainly hemorrhagic complications and atrial fibrillation. ${ }^{4,9,10}$ Bleeding, particularly skin-mucosal hemorrhagic diathesis, was found in $20-40 \%$ of patients. ${ }^{4,8-10}$ Atrial fibrillation was the $2^{\text {nd }}$ unexplained and potentially dangerous side effect, found in $3.5-6.5 \%$ of patients treated with ibrutinib (3 times more frequently than in the placebo group). ${ }^{4,9,10}$

Despite the ability of ibrutinib to induce durable responses in the vast majority of patients along with good treatment tolerance, some patient groups treated within clinical trials have had to discontinue their treatment due to disease progression or drug side effects. Recently, it has been reported that those patients have a very poor outcome. ${ }^{11,12}$ Understanding the potential reasons for discontinuing a new therapy is vital in clinical practice. Our study has therefore analyzed the causes for discontinuing ibrutinib and the subsequent course of the disease in relapsed or refractory CLL patients treated according to the ibrutinib compassionate use program and monitored within the observational study of the Polish Adult Leukemia Study Group (PALG).

\section{Material and methods}

\section{Patients}

Polish CLL patients treated within a compassionate ibrutinib use program for relapsed or refractory CLL and monitored within observational study by PALG ${ }^{13}$ were included if they discontinued ibrutinib for any reason. The inclusion criteria to the compassionate ibrutinib use program for relapsed or refractory CLL patients in Poland were at least one of the following: a) the deletion of the short-arm of chromosome 17 (17p deletion); b) failure of 2 or more previous treatments (at least 1 with a purine analogue such as fludarabine); c) a progression-free interval of less than 24 months from completing treatment with a nucleoside analogue or a bendamustine-containing regimen in combination with an anti-CD20 monoclonal antibody such as rituximab; d) failure to respond to prior chemotherapy-based treatment; e) stable disease or disease progression on treatment; and f) ineligibility for treatment or re-treatment with a purine analogue-based therapy. The prospective observational study assessing ibrutinib efficacy and toxicity in CLL patients treated in the compassionate use program was designed by PALG and approved by the Ethics Committee at the Institute of Hematology and Transfusion Medicine in Warszawa, Poland. ${ }^{13}$ The study was conducted in accordance with the provisions of the Declaration of Helsinki and the International Conference on Harmonization Guidelines for Good Clinical Practice.

The clinical data on the course of ibrutinib treatment was collected anonymously using electronic Case Report Forms (CRFs). The causes of discontinuation of ibrutinib as reported by treating physicians were analyzed. Patients who died while continuing ibrutinib therapy were also included in the analysis irrespective of the cause of death. The criteria of the National Cancer Institute Common Terminology Criteria for Adverse Events v. 4.0 were used to grade toxicity, except for hematologic toxicity cases which were evaluated using the International Workshop Group on CLL (IWCLL) criteria. ${ }^{14}$

\section{Statistical analysis}

The duration of ibrutinib therapy was measured from the starting date of ibrutinib to the date of discontinuing ibrutinib. The overall survival (OS) plots were generated according to the Kaplan-Meier method using time intervals from the date of discontinuing ibrutinib to the date of death or last follow-up. The log-rank test was used to compare survival in groups of patients discontinuing ibrutinib for 
different reasons. A p-value below 0.05 was considered statistically significant. All analyses were performed with Prism v. 6.0 software (GraphPad, La Jolla, USA).

\section{Results}

With a data cut-off set at June 2017, 37 (22.4\%) of 165 patients monitored in the PALG observational study discontinued compassionate use ibrutinib therapy. The current median follow-up time in the observational study was 26 months. The discontinuation group consisted of 22 male and 15 female patients with a median age of 64 years (range: 46-84). The median time of ibrutinib treatment in this group was 4.4 months (range: 0.2-25.2). Baseline characteristics of these patients at the time of starting ibrutinib and response to ibrutinib are presented in Table 1. Importantly, fluorescent in situ hybridization (FISH) cytogenetics was performed in 20 (65\%) patients, of whom the $17 \mathrm{p}$ deletion was found in 7 patients (19\% of the whole group) as compared to 30 patients $(18.4 \%)$ in the whole study.

Table 1. Baseline characteristics of the 37 patients analyzed at the time of the start of ibrutinib therapy

\begin{tabular}{|c|c|}
\hline Characteristic & Number of patients \\
\hline Median age (range) & $64(46-84)$ \\
\hline Male/female sex (ratio) & $22 / 15(1.47)$ \\
\hline $\begin{array}{l}\text { ECOG performance status } \\
0 \\
1 \\
2 \\
3 \\
4\end{array}$ & $\begin{array}{c}1(2.7 \%) \\
16(43.3 \%) \\
13(35.1 \%) \\
4(10.8 \%) \\
3(8.1 \%)\end{array}$ \\
\hline $\begin{array}{l}\text { CLL clinical stage (according to Rai } \\
\text { classification) } \\
0 \\
\text { I } \\
\text { II } \\
\text { III } \\
\text { IV }\end{array}$ & $\begin{array}{c}0(0 \%) \\
8(22 \%) \\
8(22 \%) \\
6(16 \%) \\
15(40 \%)\end{array}$ \\
\hline $\begin{array}{l}\text { 17p deletion by FISH } \\
\text { present } \\
\text { absent } \\
\text { not tested }\end{array}$ & $\begin{array}{l}7(19 \%) \\
13(46 \%) \\
17(35 \%)\end{array}$ \\
\hline $\begin{array}{l}\text { Previous therapies } \\
\text { Median (range) } \\
\geq 3 \text { lines of therapy }\end{array}$ & $\begin{array}{c}3(1-10) \\
18(48.6 \%)\end{array}$ \\
\hline $\begin{array}{l}\text { Duration of ibrutinib therapy } \\
\text { Median (range) }\end{array}$ & 4.4 months $(0.2-25.2)$ \\
\hline $\begin{array}{l}\text { Best response during therapy with } \\
\text { ibrutinib } \\
\text { CR } \\
\text { PR } \\
\text { PR with lymphocytosis } \\
\text { SD } \\
\text { PD }\end{array}$ & $\begin{array}{c}1(2.9) \\
11(31.4) \\
5(14.3) \\
17(48.6) \\
1(2.9)\end{array}$ \\
\hline
\end{tabular}

ECOG - Eastern Cooperative Oncology Group; CLL - chronic lymphocytic leukemia; FISH - fluorescent in situ hybridization; CR - complete response; PR - partial response; SD - stable disease; PD - progressive disease.
Table 2. Reasons for discontinuing ibrutinib therapy in the compassionate use program in Poland

\begin{tabular}{|l|c|}
\multicolumn{1}{|c|}{ Cause for discontinuing ibrutinib } & $\begin{array}{c}\text { Number } \\
\text { of patients } \\
{[\%]}\end{array}$ \\
\hline Adverse events & $20(54)$ \\
\hline Pneumonia & $6(16.2)$ \\
Upper respiratory tract infection & $3(8.1)$ \\
Diarrhea & $2(5.4)$ \\
Heart failure & $2(5.4)$ \\
Bladder cancer & $1(2.7)$ \\
Exacerbation of chronic obstructive pulmonary disease & $1(2.7)$ \\
Neutropenia & $1(2.7)$ \\
Hyphema & $1(2.7)$ \\
Suicide (severe episode of depression) & $1(2.7)$ \\
Vomiting & $1(2.7)$ \\
Cerebellar ischemic stroke & $1(2.7)$ \\
\hline Progressive disease & $14(37.8)$ \\
\hline Progression of chronic lymphocytic leukemia & $11(29.7)$ \\
Richter's transformation & $3(8.1)$ \\
\hline Patient decision & $2(5.4)$ \\
\hline Unknown (patient lost to follow-up) & $1(2.7)$ \\
\hline
\end{tabular}

The main reasons for discontinuing ibrutinib included adverse events $(n=20,54 \%)$ and disease progression ( $\mathrm{n}=14,38 \%$ ), while 3 patients $(8 \%)$ stopped treatment due to other causes (Table 2). Treatment adverse events predominated as being the cause for ibrutinib therapy cessation and consisted of: pneumonia, upper respiratory tract infection, diarrhea, vomiting, neutropenia, hyphema, bladder cancer, heart failure, exacerbation of chronic obstructive pulmonary disease, ischemic cerebellar stroke, and a suicide committed during an episode of major depression (Table 2). The most common adverse event leading to ibrutinib cessation was severe pneumonia observed in 6 patients or $16 \%$ of the discontinuation group. The etiology of pneumonia was identified in only 3 patients, while in the remainder blood culture tests were negative or not performed. Among patients with known etiology, there were 2 cases of pneumonia of bacterial origin (Acinetobacter baumani and Pseudomonas aeruginosa) and 1 case with highly probable fungal infection (Candida glabrata detected in blood culture and the gastrointestinal tract).

In those patients who progressed while on ibrutinib therapy, 2 types of progression could be identified: progression of untransformed CLL and transformation to high grade lymphoma (Richter's transformation - RT). Interestingly, 2 of the patients who had untransformed CLL experienced rare infiltration of CLL into the central nervous system (CNS) that was confirmed with a biopsy. The $1^{\text {st }}$ patient with CNS involvement died 1 day after discontinuing ibrutinib due to pneumonia and progressive disease. The $2^{\text {nd }}$ patient with CNS disease failed to respond to further therapy with high-dose methylprednisolone and died 8 months after his ibrutinib therapy was terminated. In the remaining 9 patients with CLL progression without clinical signs of CNS involvement, only 3 received subsequent lines of therapy while a palliative approach 
was chosen for the other 6 because of poor general status. Of the former 3 treated patients, 1 achieved partial response after 5 cycles of R-CHOP chemotherapy and has remained alive without signs of disease progression within a follow-up of 7 months. The $2^{\text {nd }}$ patient received 1 cycle of chemoimmunotherapy with rituximab, gemcitabine, carboplatin, and dexamethasone with no response, and then was treated with an inhibitor of 3-phospoinositol kinase idelalisib. Therapy with idelalisib did not, however, prove effective and the patient died due to progressive disease 2 weeks later. The last patient first received 4 cycles of the anti-CD20 monoclonal antibody obinutuzumab in monotherapy with no response. As the second line of treatment, CHOP chemotherapy was administered, but the patient also failed to respond and died 3 months from the day of discontinuing ibrutinib due to CLL progression. Importantly, none of the patients progressing with CLL received venetoclax, as this drug was not available.

Richter's transformation was observed in 3 patients. Interestingly, in all these 3 cases the rare type of transformation to Hodgkin's lymphoma was diagnosed. Two had transformed within the first 4 months of starting ibrutinib. One was treated according to the ABVD chemotherapy regimen but with no response. In the $2^{\text {nd }}$ patient, treatment was not initiated because of poor general condition. Both patients died within 6 months after discontinuing ibrutinib therapy. The $3^{\text {rd }}$ patient with RT, diagnosed 20 months from starting ibrutinib, has remained alive in remission after high-dose immunochemotherapy and allogeneic stem cell transplantation (alloSCT) at 13 months from discontinuing ibrutinib. A more detailed description of these rare case series is reported elsewhere.${ }^{15}$ Regarding the 3 patients who discontinued ibrutinib for other reasons than CLL progression or adverse events, in 2 the cause was the patient's decision, while in 1 patient the reason for discontinuation was unknown, the patient being lost from follow-up.

By the data cut-off for this report (June 2017), 29 (78.4\%) out of 37 patients who discontinued ibrutinib died and 8 have remained alive. The median OS is 2.0 months (95\% CI $=0.8-5.5)$ (Fig. 1). The major causes of death were disease progression and infections. All mortality causes are summarized in Table 3. In order to further investigate the potential differences in outcomes of specific patient groups, we compared the survival of patients after discontinuing ibrutinib due to disease progression (untransformed CLL progression or RT) with that of patients who discontinued ibrutinib due to adverse events or other reasons. Interestingly, the median survival was comparable in these 2 patient groups, being respectively 2.0 months (range: 0.2-10.4) and 1.6 months (range: $0.2-16.7$ ) ( $\mathrm{p}=0.81$ ). (Fig. 2). Furthermore, no significant differences could be found regarding OS of patients who progressed with CLL as compared to those with RT type of progression, although the case numbers are very low (Fig. 3).

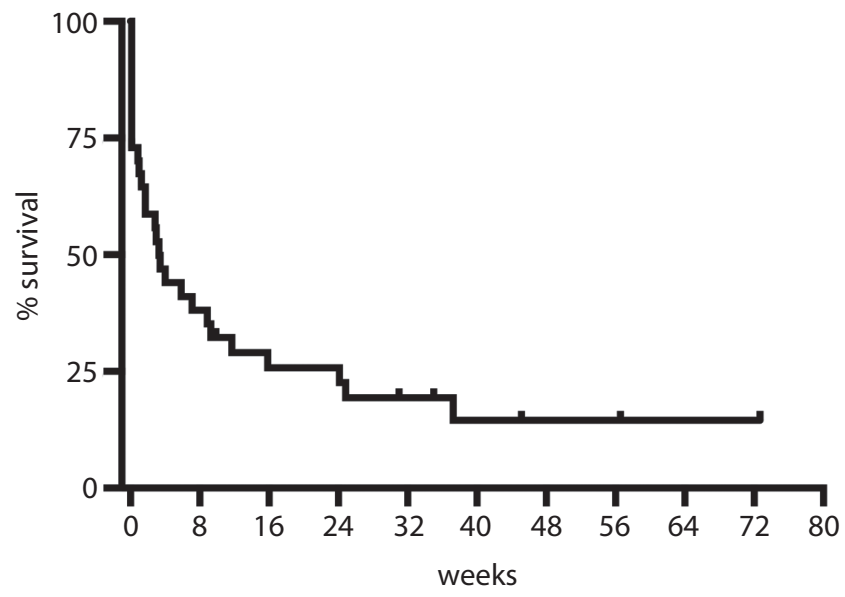

Fig. 1. Kaplan-Meier plot representing the overall survival of all patients who had to discontinue therapy with ibrutinib

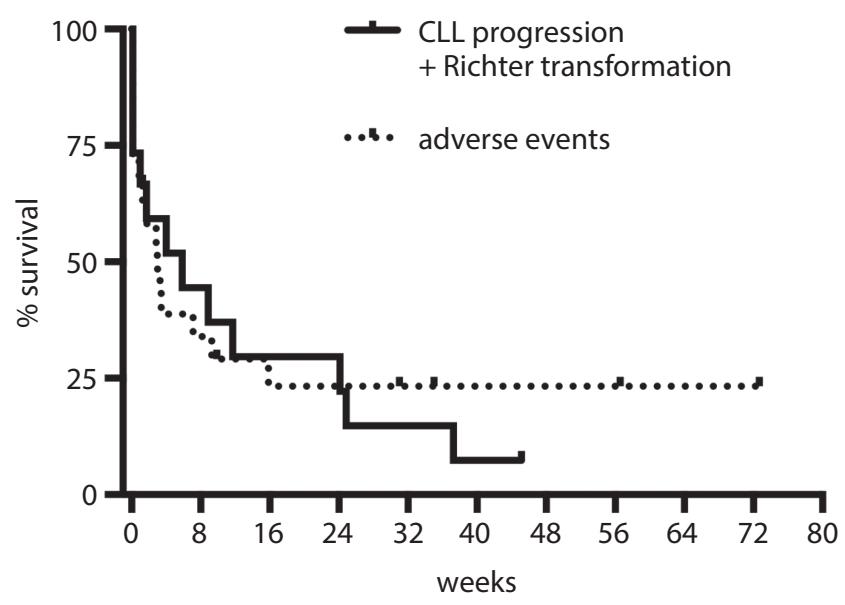

Fig. 2. Comparisons of overall survival in patients after discontinuing ibrutinib due to disease progression with that of patients who discontinued ibrutinib due to adverse events or other reasons. Median survival was comparable in these 2 patients groups, being respectively 2.0 months (range: $0.2-10.4)$ and 1.6 months (range: $0.2-16.7)(p=0.81)$

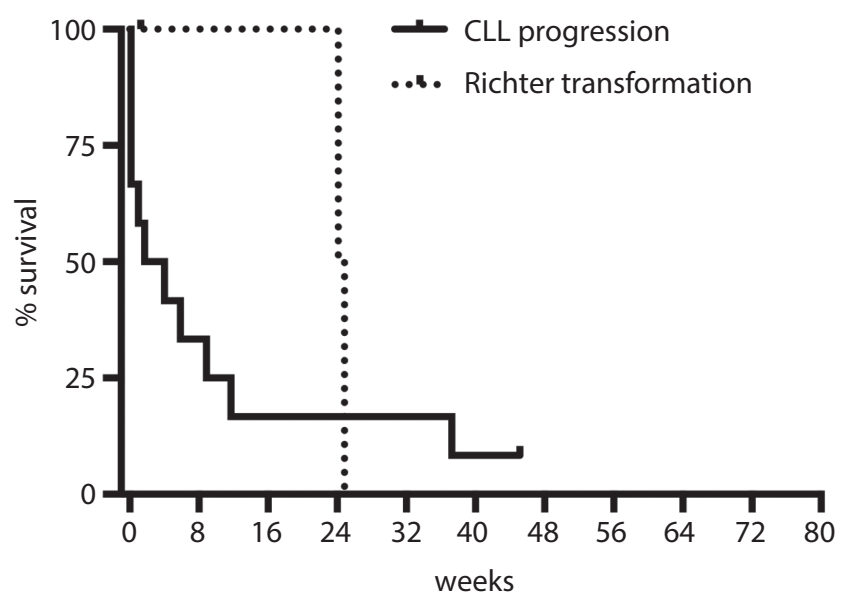

Fig. 3. Survival curves indicate no significant differences in the OS of patients with progression of chronic lymphocytic leukemia as compared to those with a Richter's transformation type of progression $(p=0.34)$ 
Table 3. Causes of death in the CLL patient group studied after ibrutinib discontinuation

\begin{tabular}{|l|c|}
\multicolumn{1}{|c|}{ Cause of death $(\mathrm{n}=29)$} & Number of patients \\
\hline Progression of CLL & 10 \\
\hline Infections & 7 \\
\hline Multi-organ failure & 3 \\
\hline Heart failure & 3 \\
\hline Gastrointestinal bleeding & 1 \\
\hline Cerebellar ischemic stroke & 1 \\
\hline Exacerbation of chronic obstructive pulmonary & 1 \\
disease & 1 \\
\hline Suicide during severe episode of depression & 1 \\
\hline Bladder cancer & 1 \\
\hline Unknown & \\
\hline
\end{tabular}

\section{Discussion}

Knowledge of the patterns of a new anti-cancer therapy discontinuation, including the rate and type of disease progression as well as a profile of severe adverse events, is very important for clinical practice. Herein, we report the outcomes of 37 relapsed or refractory CLL patients who discontinued ibrutinib therapy outside clinical trials. Our analysis confirms a very poor prognosis of such a patient group irrespective of whether the cause of discontinuation was relapse or transformation of CLL or ibrutinib treatment-related complications.

Early reports from clinical trials indicate that the main reasons for discontinuing ibrutinib treatment were severe adverse events and/or confirmation of progressive disease. In a $1 b-2$ phase, multicenter and open label study, the investigators assessed the safety, efficacy, pharmacokinetics, and pharmacodynamics of ibrutinib ${ }^{7}$ which showed that BTK inhibitor therapy had to be halted in $37 \%$ of patients. Among the prevailing reasons were progressive disease (13\%) and adverse events (8\%). Subsequently, 2 reports from single institutions specifically analyzed patient outcomes for those treated with ibrutinib in different clinical trials and who had to discontinue therapy. The $1^{\text {st }}$ report presented 33 patients participating in various clinical trials with ibrutinib alone or combined with rituximab at the MD Anderson Cancer Center (Houston, USA). ${ }^{11}$ In the $2{ }^{\text {nd }}$ one, Maddocks et al. ${ }^{12}$ described 76 patients treated in 4 sequential ibrutinib clinical trials at the Ohio State University Comprehensive Cancer Center (USA). These reports showed that the main reasons for discontinuing ibrutinib were adverse events and disease progression. The most common side effects responsible for discontinuing ibrutinib were severe infections. An increased number of RT was also observed in such cases. ${ }^{11,12}$

Our analysis demonstrated that $22.4 \%$ (37 out of 165) of patients treated within the compassionate use ibrutinib program discontinued therapy after a median follow-up of 26 months. These findings are comparable to the reports on patients treated in clinical trials, where ibrutinib was discontinued in $26 \%$ and $24.6 \%$ of patients, respectively. ${ }^{11,12}$ Also consistently with these reports, ${ }^{11,12,16}$ the most common reason for ibrutinib cessation was adverse events (54\% of patients), where severe infections predominated. Notwithstanding, the Jain et al. study found that the median duration of ibrutinib treatment was significantly longer (13 months vs 4.4 months) than in our findings with no obvious differences in patient characteristics. ${ }^{11}$ This could be partially explained by the fact that physicians exercise excessive caution to adverse events when using a new drug outside of clinical trials, as in the case of our report. The cause of severe infections associated with ibrutinib treatment in CLL patients is most probably multifactorial, involving pre-existing immune-suppression associated with the biological mechanisms of CLL and drug-induced susceptibility to infection. ${ }^{17-19}$ Interestingly, the results of a recent meta-analysis concerning infectious complications of ibrutinib therapy for hematological malignancies have indicated a significant association between exposure to this drug and an increased risk of serious infections. ${ }^{19}$ Therefore, carefully observing fever and other signs and symptoms of infection together with monitoring for neutropenia and searching for potentially responsible pathogens would enable an appropriate anti-infective therapy to be introduced early. Recognizing which CLL patients treated with BTK inhibitors are at a higher risk of developing infectious adverse events could be an important future challenge.

Although not occurring in our study group, a potential reason for discontinuation could also be atrial fibrillation. ${ }^{20}$ It should therefore be stressed that discontinuing treatment in patients who develop atrial fibrillation when on ibrutinib therapy is not always necessary. In such situations, a thorough risk assessment for thromboembolic disease should be undertaken. In patients at high risk and where alternatives to ibrutinib are unsuitable, a strictly controlled treatment with anticoagulants should be considered. ${ }^{21}$ Appropriate management of adverse events could thereby prevent premature discontinuation of therapy.

Disease progression was the reason for discontinuation in 14 (38\%) of our patients including 8 patients (30\%) with CLL progression and 3 patients $(8 \%)$ with RT. It has been found that disease progression in CLL patients treated with ibrutinib has been attributed to the clonal evolution of CLL with acquired mutations in BTK (substitution of cysteine 481 with serine) and phospholipase C- $\gamma 2$ (PLCG2) (S707Y, R6 65W and L845F) or histologic transformation (RT). ${ }^{22,23}$ Unfortunately, we did not assess the occurrence of resistance mutation because the biologic material from the time of relapse was unavailable.

Interestingly, the infiltrations of CLL into the CNS were observed in 2 patients. Central nervous system involvement is a rare complication in CLL and displays heterogeneous neurological symptoms. ${ }^{24}$ The therapy for this rare event is still undetermined. Wanquet et al. observed good responses in patients with CLL infiltration of the CNS who were 
treated with ibrutinib (5-year OS was respectively $72 \%$ and $48 \%$ for treatment-naïve and previously treated patients). ${ }^{24}$ Patients with relapsed or refractory CLL and confirmed CNS involvement during ibrutinib therapy had limited therapeutic options. However, prognosis seemed to be related to CLL characteristics rather than to the CNS infiltration itself. ${ }^{24}$

The $2^{\text {nd }}$ type of progression that was observed in 3 of our patients was RT, which is generally found in about $2-10 \%$ of CLL patients. ${ }^{25}$ Interestingly, it was reported that in patients treated with ibrutinib who progressed, RT may be particularly common. ${ }^{23}$ It is still unclear if ibrutinib increases the risk of RT or if patients included in ibrutinib therapy constitute groups with poorer prognoses than the general population suffering from CLL. Current observations indicate that during ibrutinib therapy, progressive CLL occurred later than RT. In the Ahn et al. study, histologic transformation was limited to the first 15 months when on ibrutinib while progression due to CLL was diagnosed at a median of 38 months. ${ }^{26}$ Maddocks et al. ${ }^{12}$ likewise found the estimated rates of cumulative $\mathrm{RT}$ incidence at 12 months to be $4.5 \%$ (95\% CI = 2.0-7.0\%) and the estimated rates of the cumulative incidence of progressive disease due to CLL at 12 months to be $0.3 \%$ (95\% $\mathrm{CI}=0-1.0 \%)$. Our results are thus comparable, with 2 out of 33 patients with RT diagnosed in the $4^{\text {th }}$ month of ibrutinib therapy. In contrast to the majority of RT cases under ibrutinib, the RT in the $3^{\text {rd }}$ patient was diagnosed after almost 2 years of ibrutinib treatment.

Interestingly, in our analysis, all 3 RT patients were diagnosed with a Hodgkin variant of RT, a rare form accounting for $0.4-2.3 \%$ of CLL cases ${ }^{27}$ However, 2 recent case reports on patients with the Hodgkin variant of RT during ibrutinib therapy have been published. ${ }^{28,29}$ Their outcomes of therapy are superior to those of the more common RT in diffusing large B-cell lymphoma. ${ }^{30}$ Nevertheless, patients with Hodgkin's lymphoma and RT who had received purine nucleoside analogs for CLL have very short survivals if treated with HL-type therapy. ${ }^{31,32}$ Interestingly, one of our RT patients responded favorably to intensive chemotherapy consolidated with alloSCT and remains alive at 13 months after transplantation.

Our data suggests that the survival of relapsed/refractory CLL patients who discontinued ibrutinib is poor. The median OS was 2.0 months $(95 \% \mathrm{CI}=0.8-5.5)$ and did not differ significantly between patients who progressed and those who discontinued ibrutinib due to other causes. The results of available studies also confirm the unfavorable outcomes of this category of CLL patients. ${ }^{11,12,16}$ However, in contrast to our findings, the largest study from the MD Anderson Cancer Center indicated that survival was significantly influenced by the reason of ibrutinib discontinuation. Patients who had progressive CLL had better survival compared with those who had disease transformation. ${ }^{16}$ Additionally, Jain et al. suggested that one of the possible reasons for the poor salvage ability of patients who discontinued ibrutinib was a higher proportion of patients having 17 p deletion (58\% vs $25 \%$ ) and unmutated IGHV (94\% vs 74\%) compared with patients who continued receiving ibrutinib therapy. ${ }^{11}$ Also, our group contained a relatively high proportion of $17 \mathrm{p}$ deleted patients (7 out of 20 tested patients, 35\%), but unfortunately the IGHV mutational status was not examined in any of the patients included.

Importantly, recent studies on new therapeutic options for this patient group have been performed. Patients who discontinued ibrutinib due to progression or toxicity had improved outcomes if they received venetoclax (ORR $79 \%$ ) or idelalisib (ORR 46\%) compared to immunochemotherapy. ${ }^{33}$ Venetoclax, a selective BCL-2 inhibitor, was approved by the Food and Drug Administration in the USA in April 2016 for patients with previously treated del17p CLL on the basis of a single-arm, multicenter, phase II trial. ${ }^{33}$ Based on the positive results of subsequent studies, extension for indicating its use is to be expected. ${ }^{34-37} \mathrm{Un}$ fortunately, our patients did not have access to venetoclax.

Our analysis has confirmed that discontinuing ibrutinib therapy in patients with relapsed or refractory CLL is associated with poor prognosis. Elucidating the biological mechanisms of resistance in CLL as well as investigating new targeted therapeutic options such as venetoclax, are thus needed to improve treatment outcomes for this patient group.

\section{References}

1. Swerdlow SH, Campo E, Harris NL, et al. WHO Classification of Tumours of Haematopoietic and Lymphoid Tissues. $4^{\text {th }}$ ed. Lyon, France: International Agency for Research on Cancer; 2008.

2. Pulte D, Redaniel MT, Bird J, Jeffreys M. Survival for patients with chronic leukemias in the US and Britain: Age-related disparities and changes in the early $21^{\text {st }}$ century. Eur J Haematol. 2015;94(6):540-545.

3. Eichhorst B, Robak T, Montserrat E, et al; ESMO Guidelines Committee. Chronic lymphocytic leukaemia: ESMO Clinical Practice Guidelines for diagnosis, treatment and follow-up. Ann Oncol. 2015;26(Suppl 5): 78-84.

4. Byrd JC, Brown JR, O'Brien S, et al; for the RESONATE Investigators. Ibrutinib versus ofatumumab in previously treated chronic lymphoid leukemia. N Engl J Med. 2014;371:213-223.

5. Burger JA, Chiorazzi N. B cell receptor signaling in chronic lymphocytic leukemia. Trends Immunol. 2013;34(12):592-601.

6. Ponader S, Chen SS, Buggy JJ, et al. The Bruton tyrosine kinase inhibitor $\mathrm{PCl}-32765$ thwarts chronic lymphocytic leukemia cell survival and tissue homing in vitro and in vivo. Blood. 2012;119(5):1182-1189.

7. Byrd JC, Furman RR, Coutre SE, et al. Targeting BTK with ibrutinib in relapsed chronic lymphocytic leukemia. NEngl J Med. 2013;369(1): 32-42.

8. Byrd JC, Furman RR, Coutre SE, et al. Three-year follow-up of treatment-naive and previously treated patients with CLL and SLL receiving single-agent ibrutinib. Blood. 2015;125(16):2497-2506.

9. Chanan-Khan A, Cramer P, Demirkan F, et al; HELIOS Investigators. Ibrutinib combined with bendamustine and rituximab compared with placebo, bendamustine, and rituximab for previously treated chronic lymphocytic leukaemia or small lymphocytic lymphoma [HELIOS]: A randomised, double-blind, phase 3 study. Lancet Oncol. 2016;17(2):200-211.

10. Burger JA, Tedeschi A, Barr PM, et al; for the RESONATE-2 Investigators. Ibrutinib as initial therapy for patients with chronic lymphocytic leukemia. N Engl J Med. 2015;373:2425-2437.

11. Jain $P$, Keating $M$, Wierda W, et al. Outcomes of patients with chronic lymphocytic leukemia after discontinuing ibrutinib. Blood. 2015; 125(13):2062-2067. 
12. Maddocks KJ, Ruppert AS, Lozanski G, et al. Etiology of ibrutinib therapy discontinuation and outcomes in patients with chronic lymphocytic leukemia. JAMA Oncol. 2015;1(1):80-87.

13. Iskierka-Jażdżewska E, Hus M, Giannopoulos K, et al. Efficacy and toxicity of compassionate ibrutinib use in relapsed/refractory chronic lymphocytic leukemia in Poland: Analysis of the Polish Adult Leukemia Group (PALG). Leuk Lymphoma. 2017:58(10):2485-2488.

14. Hallek M, Cheson BD, Catovsky D, et al; International Workshop on Chronic Lymphocytic Leukemia. Guidelines for the diagnosis and treatment of chronic lymphocytic leukemia: A report from the International Workshop on Chronic Lymphocytic Leukemia updating the National Cancer Institute-Working Group 1996 guidelines. Blood. 2008;111(12):5446-5456.

15. Jamroziak K, Szymczyk A, Hus M, et al. A series of chronic lymphocytic leukemia patients with Hodgkin variant Richter's transformation during ibrutinib therapy: A report from the Polish Adult Leukemia Group (PALG). Eur J Haematol. 2018. doi: 10.1111/ejh.13016

16. Jain $P$, Thompson PA, Keating $M$, et al. Long-term outcomes for patients with chronic lymphocytic leukemia who discontinue ibrutinib. Cancer. 2017;123(12):2268-2273.

17. Williams AM, Baran AM, Meacham PJ, et al. Analysis of the risk of infection in patients with chronic lymphocytic leukemia in the era of novel therapies. Leuk Lymphoma. 2018;59(3):625-632.

18. Winqvist $M$, Palma $M$, Heimersson $K$, et al. Dual targeting of Bruton tyrosine kinase and CD52 induces minimal residual disease-negativity in the bone marrow of poor-prognosis chronic lymphocytic leukaemia patients but is associated with opportunistic infections: Results from a phase I study. Br J Haematol. 2018;182(4):590-594.

19. Tillman BF, Pauff JM, Satyanarayana G, Talbott M, Warner JL. Systematic review of infectious events with the Bruton tyrosine kinase inhibitor ibrutinib in the treatment of hematologic malignancies. Eur J Haematol. 2018;100(4):325-334.

20. Thorp BC, Badoux X. Atrial fibrillation as a complication of ibrutinib therapy: Clinical features and challenges of management. Leuk Lymphoma. 2018;59(2):311-320.

21. Chai KL, Rowan G, Seymour JF, Burbury K, Carney D, Tam CS. Practical recommendations for the choice of anticoagulants in the management of patients with atrial fibrillation on ibrutinib. Leuk Lymphoma. 2017;58(12):2811-2814.

22. Furman RR, Cheng $S$, Lu $P$, et al. Ibrutinib resistance in chronic lymphocytic leukemia. N Engl J Med. 2014;370(24):2352-2354.

23. Sharma S, Galanina N, Guo A, et al. Identification of a structurally novel BTK mutation that drives ibrutinib resistance in CLL. Oncotarget. 2016;7(42):68833-68841.
24. Wanquet A, Birsen R, Bonnet $C$, et al. Management of central nervous system involvement in chronic lymphocytic leukaemia: A retrospective cohort of 30 patients. Br J Haematol. 2017;176(1):37-49.

25. Jamroziak K, Tadmor T, Robak T, Polliack A. Richter syndrome in chronic lymphocytic leukemia: Updates on biology, clinical features and therapy. Leuk Lymphoma. 2015;56(7):1949-1958.

26. Ahn IE, Underbayev C, Albitar A, et al. Clonal evolution leading to ibrutinib resistance in chronic lymphocytic leukemia. Blood. 2017; 129(11):1469-1479.

27. Tsimberidou AM, O'Brien S, Kantarjian HM, et al. Hodgkin transformation of chronic lymphocytic leukemia: The M.D. Anderson Cancer Center experience. Cancer. 2006;107(6):1294-1302.

28. Glavey S, Quinn J, McCloy M, et al. Emergence of Bruton's tyrosine kinase-negative Hodgkin lymphoma during ibrutinib treatment of chronic lymphocytic leukaemia. Eur J Haematol. 2017;99(4):378-380.

29. Sachanas S, Pangalis GA, Moschogiannis M, et al. Hodgkin lymphoma transformation of chronic lymphocytic leukemia under ibrutinib therapy: Chance association or therapy-related? Anticancer Res. 2017;37(6):3277-3280.

30. Jamroziak K, Grzybowska-Izydorczyk O, Jesionek-Kupnicka D, GoraTybor J, Robak T. Poor prognosis of Hodgkin variant of Richter transformation in chronic lymphocytic leukemia treated with cladribine. Br J Haematol. 2012;158(2):286-288.

31. Brecher M, Banks PM. Hodgkin's disease variant of Richter's syndrome: Report of eight cases. Am J Clin Pathol. 1990;93(3):333-339.

32. Bockorny B, Codreanu I, Dasanu CA. Hodgkin lymphoma as Richter transformation in chronic lymphocytic leukaemia: A retrospective analysis of world literature. Br J Haematol. 2012;156(1):50-66.

33. Mato AR, Hill BT, Lamanna N, et al. Optimal sequencing of ibrutinib, idelalisib, and venetoclax in chronic lymphocytic leukemia: Results from a multicenter study of 683 patients. Ann Oncol. 2017;28(5):10501056.

34. Stilgenbauer S, Eichhorst B, Schetelig J, et al. Venetoclax in relapsed or refractory chronic lymphocytic leukaemia with 17p deletion: A multicentre, open-label, phase 2 study. Lancet Oncol. 2016;17(6):768-778.

35. Seymour JF, Ma S, Brander DM, et al. Venetoclax plus rituximab in relapsed or refractory chronic lymphocytic leukaemia: A phase 1b study. Lancet Oncol. 2017;18(2):230-240.

36. Roberts AW, Davids MS, Pagel JM, et al. Targeting BCL2 with Venetoclax in relapsed chronic lymphocytic leukemia. N Engl J Med. 2016; 374(4):311-322.

37. Jones JA, Wierda WG, Choi MY, et al. Venetoclax activity in CLL patients who have relapsed after or are refractory to ibrutinib or idelalisib. J Clin Oncol. 2016 34(15 Suppl):7519-7519. 